\title{
SELF-ASSEMBLY DI DNA SU SUPERFICIE: UNO STUDIO COMBINATO NANOLITOGRAFIA/SPETTROSCOPIA OTTICA
}

\author{
ORNELLA CAVALLERI (*), et al. ${ }^{*}$ \\ Nota presentata dal m.e. Giorgio Benedek \\ (Adunanza del 24 ottobre 2019)
}

\begin{abstract}
SuNTO. - Il self-assembly di molecole organiche e biomolecole su superfici inorganiche è un processo complesso che deriva dal bilancio di diverse interazioni: molecola/molecola, molecola/superficie, molecola/solvente. L'interesse per lo studio del self-assembly molecolare deriva dalla possibilità di realizzare superfici funzionalizzate che presentano le proprietà funzionali proprie delle (bio) molecole depositate. I SAM (Self-Assembled Monolayers) trovano infatti ampia applicazione nel biosensing. Tra i SAM più studiati figurano i SAM di organosolfuri su oro. Nel seguito viene presentato un approccio che combina la microscopia a scansione di sonda e la spettroellissometria per lo studio delle proprietà morfologiche, meccaniche ed ottiche di SAM costituiti da filamenti di DNA, un sistema ampiamente studiato poiché, sfruttando l'ibridazione tra filamenti di DNA complementari, è alla base della realizzazione dei microarray di DNA.
\end{abstract}

$$
* * *
$$

ABSTRACT. - The self-assembly of organic molecules and biomolecules on inorganic surfaces is a complex process which results from the interplay between different interactions: molecule/molecule, molecule/surface, and molecule/solvent interactions. The interest in the study of molecular self-assembly derives from the possibility to obtain functionalized surfaces that exhibit the functional properties of the deposited (bio) molecules. Self-Assembled Monolayers (SAMs) are indeed widely used in biosensing.

(*) Dipartimento di Fisica, Università degli Studi di Genova, Italy.

E-mail: cavalleri@fisica.unige.it

\# La nota è il risultato di un lavoro di ricerca condotto in collaborazione con: Giulia Pinto e Maurizio Canepa. 
SAMs of organosulphur compounds on gold are among the most widely studied SAMs In the following, a combined approach that couples scanning probe microscopy and spectroellipsometry is discussed and applied to the study of the morphological, mechanical and optical properties of SAMs of DNA single strands. DNA SAMs are a widely investigated system since they can be exploited for the design of DNA microarrays based on the hybridization between complementary DNA strands.

\section{INTRODUZIONE}

A partire dagli anni ' 80 con i primi lavori di Nuzzo, Allara e Whitesides sul self-assembly di alcantioli di superfici d'oro[1-3], lo studio del self-assembly di molecole organiche su superfici ha conosciuto un enorme sviluppo. Negli anni '80-'90, sono stati portati avanti studi di carattere fondamentale indirizzati a comprendere quali siano le interazioni coinvolte nella formazione dei SAM e come queste determinino l'ordinamento molecolare all'interno dei SAM. Schematizzata la molecola come composta da un gruppo di ancoraggio alla superficie, una catena centrale e un gruppo funzionale terminale (Fig. 1), tali studi hanno messo in evidenza come la struttura dei SAM derivi essenzialmente dal bilancio di tre tipi di interazioni: le interazioni gruppo di ancoraggio/superficie, responsabili del legame, in genere covalente, del SAM al substrato, le interazioni inter-catena, essenzialmente di tipo van der Waals nel caso di semplici catene alchiliche, e le interazioni tra i gruppi funzionali terminali, la cui natura dipende dalle proprietà del gruppo terminale stesso. Il complesso bilancio di queste interazioni, modulato da fattori dipendenti dalle molecole, quali la lunghezza della catena alchilica o la natura del gruppo terminale (polarità, carica, ingombro sterico, ecc), e da fattori esterni, quali la struttura cristallografica della superficie, il tipo di solvente utilizzato per la deposizione o la temperatura, determina la densità e il grado di ordine molecolare del SAM [4]. In funzione della coppia gruppo di ancoraggio/superficie, si possono identificare due classi principali di SAM: i SAM formati da molecole con il gruppo tiolo (-SH) come gruppo di ancoraggio, che presenta un'elevata affinità per le superfici di metalli quali $\mathrm{Au}, \mathrm{Ag}, \mathrm{Pt}, \mathrm{Cu}$ o $\mathrm{Hg}$, e i SAM formati su superfici di ossidi da molecole aventi diversi gruppi di ancoraggio, tra cui silani, fosfonati o carbossilati [5]. Nel seguito ci soffermeremo sui SAM di organosolfuri su oro. 


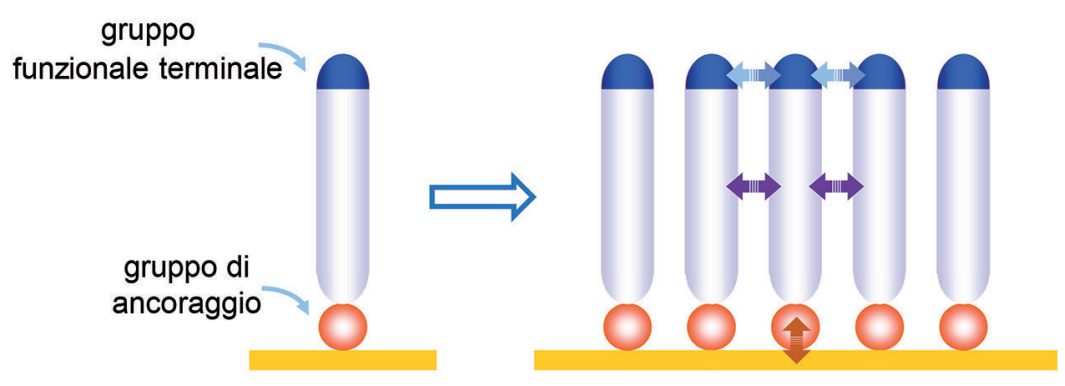

Fig. 1. Dalla molecola su superficie al SAM.

Dopo i primi studi strutturali condotti su SAM formati da molecole relativamente semplici, l'attenzione si è via via spostata verso lo studio di SAM formati da molecole con gruppi funzionali più complessi in grado di dare origine alla formazione di SAM con proprietà funzionali più interessanti da un punto di vista applicativo, dal sensing di ioni metallici [6] alla rilevazione di marker tumorali [7]. Va sottolineato che al crescere delle proprietà funzionali del gruppo terminale è associato in genere un aumento dell'ingombro sterico e della complessità delle interazioni intermolecolari che guidano il processo di self-assembly. Nel seguito discuteremo la deposizione, per self-assembly, di filamenti di DNA. SAM di singoli filamenti di oligonucleotidi sono alla base della realizzazione di microarray di DNA, ampiamente impiegati nel biosensing. La caratteristica chiave di questi dispositivi è il riconoscimento altamente specifico derivante dall'accoppiamento tra basi complemetari che può essere sfruttato per la rilevazione selettiva di diversi analiti, dagli oligonucleotidi fino alle cellule [8].

L'ibridazione tra sequenze complementari può essere sfruttata per il biosensing su due livelli. Da un lato, può essere utilizzata per il riconoscimento diretto di sequenze target specifiche, con applicazioni in biologia molecolare e nella diagnostica, poiché alcune patologie possono essere identificate sulla base della rilevazione di specifiche sequenze di DNA o RNA. Esempi ne sono l'identificazione di S. Aureus [9], di sequenze di mi-RNA correlate a tumori o malattie cardiovascolari $[10,11]$ o di sequenze di RNA di virus [12,13]. D'altra parte, l'ibridazione può anche essere sfruttata per l'immobilizzazione su superficie di molecole sensori che mantengano le loro proprietà selettive e funzionali anche dopo l'immobilizzazione: in questo caso, l'ibridazione tra la sequenza di DNA ancorata alla superficie e la sua sequenza complementare, opportuna- 
mente funzionalizzata con la molecola sensore, permette di ancorare quest'ultima alla superficie. In questo modo, le molecole sensore (ad esempio anticorpi) possono essere ancorate alla superficie, pur rimanendo disaccoppiate da essa grazie al SAM di DNA, mantenendo quindi la propria funzionalità e selettività verso specifici marcatori molecolari [14-16].

La rilevanza dei SAM di DNA per lo sviluppo di biosensori ha motivato numerosissimi studi nell'arco degli ultimi 20 anni, riguardanti il self-assembly di singoli filamenti di DNA tiolati e della loro ibridazione [17-20]. Per lo sviluppo di sensori robusti ed affidabili, è cruciale infatti procedere attraverso un'attenta caratterizzazione del monostrato di DNA. Rispetto al self-assembly di molecole più semplici come gli alcantioli, il chemisorbimento di filamenti di DNA tiolati su oro è un processo più complesso, dal momento che i filamenti di DNA non sono molecole neutre, ma hanno una carica netta negativa dovuta alla presenza dei gruppi fosfato e presentano un maggiore ingombro sterico, parametri entrambi modulabili dalla forza ionica della soluzione $[21,22]$. Tra i metodi sperimentali impiegati per studiare il self-assembly di DNA su oro figurano metodi elettrochimici $[23,24]$, così come metodi spettroscopici, dalla spettroscopia ottica [25], a quella infrarossa [26] e a raggi X [27].

\section{NANOLITOGRAFIA COMBINATA ALLA SPETTROELLISSOMETRIA PER L'ANALISI DI SAM DI DNA}

Nel seguito vengono presentati i risultati di un approccio metodologico combinato nanolitografia/spettroscopia ottica per lo studio delle proprietà morfologiche, meccaniche ed ottiche di SAM di DNA.

Un aspetto finora meno studiato dei SAM di DNA è quello che riguarda le loro proprietà meccaniche. La valutazione delle proprietà meccaniche di film ultrasottili come i SAM è un obiettivo complesso, poiché è necessaria un'elevata sensibilità per poter disaccoppiare il contributo del film da quello del substrato. In effetti, la valutazione delle proprietà meccaniche di film organici sottili ed ultrasottili è un aspetto importante per lo sviluppo e l'ottimizzazione dei biosensori, in quanto è stato mostrato che l'interazione tra substrati e campioni biologici, in particolare cellule, è fortemente influenzata dalla rigidità del substrato [28].

Come di seguito riportato, l'utilizzo della microscopia a scansione di sonda, accoppiata a metodi nanolitografici, permette di studiare le proprietà morfologiche e meccaniche di SAM di DNA ed è sufficiente- 
mente sensibile da discriminare tra SAM di DNA depositati a diverse forze ioniche.

Per correlare proprietà strutturali e proprietà ottiche dei SAM, gli esperimenti di nanolitografia sono stati accoppiati a misure di spettroellissometria (SE). L'ellissometria spettroscopica è una tecnica ottica affidabile, rapida, altamente sensibile e non perturbativa, che misura la variazione di polarizzazione di un raggio luminoso in seguito alla riflessione da una superficie, fornendo informazioni sullo spessore e le proprietà ottiche di film sottili e ultrasottili. Per film ultrasottili, come i SAM di DNA, spessore e indice di rifrazione sono parametri correlati. Tale correlazione può essere risolta mediante un'analisi combinata sfruttando i dati di spessore ottenuti dalla nanolitografia [29,30].

A partire dai primi lavori di Liu [31,32], la nanolitografia a scansione di sonda (nanoshaving e nanografting) è stata impiegata con successo per produrre interfacce organiche/inorganiche patternate per l'immobilizzazione selettiva di nano-oggetti (dalle nanoparticelle alle biomolecole) $[33,34]$ e per sviluppare piattaforme sensibili per il riconoscimento molecolare $[35,36]$.

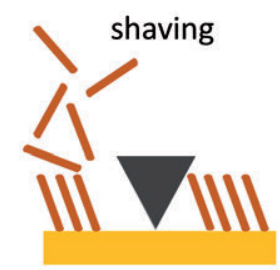

a)

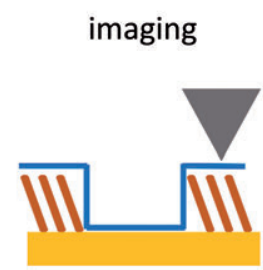

b)

Fig. 2. Rappresentazione schematica dell'utilizzo dell'AFM in modalità shaving (a) $e$ in modalità imaging (b).

Gli esperimenti di nanoshaving possono essere vantaggiosamente impiegati anche per misurare lo spessore degli strati molecolari a livello sub-nanometrico, permettendo così di monitorare le variazioni di spessore del film in seguito all'adsorbimento selettivo di analiti. In un esperimento di nanoshaving la punta del microscopio a forza atomica (Atomic Force Microscope, AFM) viene utilizzata come uno "scalpello" per rimuovere selettivamente le molecole in una regione del monostrato (Fig. 2). Modulando la forza applicata, è possibile passare da condizioni di soft imaging (forza bassa) a condizioni di shaving (forza alta). In genere gli esperimenti di shaving vengono effettuati in modalità con- 
tatto sia nella fase di imaging sia in quella di rimozione selettiva delle molecole. Una variante metodologica interessante consiste nel lavorare in modalità quantitative imaging (QI) nella fase di imaging. Il QI (https://www.jpk.com/products/atomic-force-microscopy/qi-mode) è una modalità di imaging basata sulla spettroscopia di forza: attraverso l'acquisizione di un ampio set di curve forza-distanza, consente di ricostruire la topografia del campione attraverso una mappa della posizione $z$ della punta a forza applicata fissata. Poiché la punta viene avvicinata ed allontanata dalla superficie punto per punto durante la scansione, le forze laterali sono ridotte al minimo e si evitano problemi di trascinamento. Inoltre, la combinazione di imaging e spettroscopia di forza permette di ottenere dati quantitativi non solo relativi alle misure di spessore del film, ma anche sulle sue proprietà meccaniche. In particolare, l'analisi delle curve di forza consente la valutazione locale di parametri meccanici come l'adesione e il modulo di Young [37].

In Fig. 3 vengono riportati i risultati di due esperimenti di shaving condotti su SAM di DNA tiolato su oro preparati a diversa forza ionica, bassa forza ionica (1 $\mathrm{mM} \mathrm{NaCl}$, Fig. 1a-c) ed alta forza ionica (1 M $\mathrm{NaCl}$, Fig. 1d-f). Si tratta di un oligonucleotide formato da 22 basi avente un linker tiolato costituito da una catena di esantiolo. Si rimanda a Pinto et al. [38] per i dettagli sperimentali. Entrambi shaving ed imaging sono stati eseguiti nello stesso buffer utilizzato per il self-assembly del DNA. Mentre lo shaving è stato condotto in modalità hard tapping, l'imaging è stato effettuato sia in soft contact sia in QI.

Lo spessore del SAM può essere valutato dall'analisi delle immagini misurando la differenza di altezza tra la regione esterna corrispondente al SAM e la regione centrale, corrispondente all'oro, dove il SAM è stato rimosso. In Fig. $3 a, d$ sono riportate le immagini in soft contact, mentre in Fig. 3b,e sono riportate le immagini in QI relative a shaving su SAM preparati a bassa forza ionica (sezione superiore, Fig. 3a-c) e ad alta forza ionica (sezione inferiore, Fig. 3d-f). Dai profili z delle immagini si può misurare lo spessore del SAM (Fig. 3c,f).

La sovrapposizione delle curve continue (relative alle immagini acquisite in soft contact) e tratteggiate (relative alle immagini acquisite in modalità QI) indica che la stima dell'altezza ottenuta in modalità contatto è leggermente inferiore al valore derivante dalle immagini in QI. Questa differenza si può spiegare tenendo conto delle differenze tra i due tipi di imaging: in modalità contatto, l'interazione continua tra il campione e la punta può provocare forze di trascinamento che possono 
perturbare il monostrato, determinando una sottostima dell'altezza. 亡̀ quindi necessario lavorare minimizzando per quanto possibile la forza di interazione (tipicamente $0.1 \mathrm{nN}$ ). In modalità QI, l'interazione intermittente punta-campione evita gli effetti di trascinamento e si traduce in una modalità operativa meno perturbativa. Un'analisi comparativa delle immagini ottenute in modalità soft contact e QI può pertanto aumentare l'affidabilità della misura dell'altezza del SAM. Vale la pena notare che l'accordo relativo tra i profili z ottenuti nelle modalità contatto e QI è leggermente migliore per i SAM preparati ad alta forza ioni$\mathrm{ca}$, indicando che questi film sono meno vulnerabili alla perturbazione della punta AFM. Questa osservazione è in accordo con il fatto che i SAM depositati ad alta forza ionica sono più spessi, quindi verosimilmente più densi e compatti rispetto ai SAM preparati a bassa concentrazione di sale. Da un'analisi statistica dei profili z ottenuti su diversi campioni, risultano spessori di $(1.0 \pm 0.3) \mathrm{nm}$ e $(3.3 \pm 0.5) \mathrm{nm}$ per i SAM depositati, rispettivamente, a bassa e ad elevata forza ionica.

\section{bassa forza ionica}
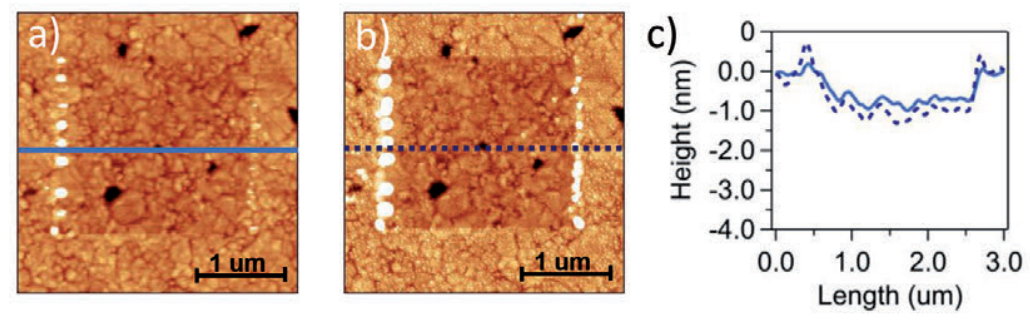

\section{alta forza ionica}
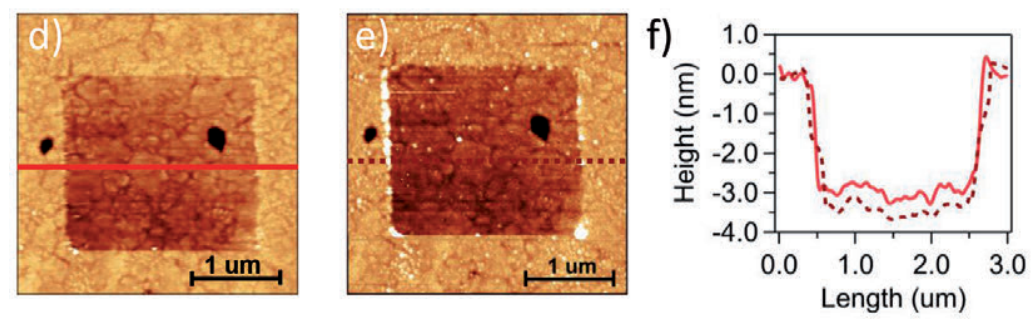

Fig. 3. Esperimenti di shaving su SAM di DNA preparati a bassa forza ionica (a-c) e ad alta forza ionica (d-f). Immagini AFM acquisite in modalità soft contact $(a, d)$ e QI $(b, e)$ (scala z: $9 \mathrm{~nm}$ ). (c,f) profili z relativi alle immagini in contatto (linee continue) e in QI (linee tratteggiate). Modificata da [38]. 
Oltre all'informazione morfologica sullo spessore, l'analisi AFM può fornire informazioni anche sulle proprietà meccaniche del SAM. La Fig. 4 riporta le mappe della deflessione laterale e del modulo di Young relativi agli esperimenti di shaving mostrati in Fig. 3. La deflessione laterale, misurata operando con l'AFM in modalità contatto, fornisce informazioni sulle proprietà tribologiche locali tra punta e campione. Ci si attende quindi di misurare diversi valori di deflessione laterale se la punta si muove su una regione dove è presente il SAM o se si muove sul substrato di oro. L'immagine in deflessione laterale (Fig. 4a) mostra una differenza molto bassa tra i valori di deflessione laterale nel caso dei SAM di DNA preparati a bassa forza ionica, mentre il contrasto è decisamente maggiore per i SAM preparati ad elevata forza ionica (Fig. 4d). Tali risultati possono essere interpretati sulla base delle misure di altezza soprariportate. I SAM preparati a bassa forza ionica hanno uno spessore molto ridotto, questo indica una densità molecolare molto bassa, verosimilmente legata ad una disposizione delle molecole quasi parallela alla superficie. Con un film di così bassa densità risulta difficile disaccoppiare le proprietà tribologiche del film da quelle del substrato sottostante. $\mathrm{Al}$ contrario, nel caso dei SAM depositati a forza ionica più elevata, che risultano più spessi e compatti, le immagini in deflessione laterale permettono di osservare una netta differenza tra SAM e substrato.

Ulteriori informazioni sulle proprietà meccaniche del campione possono essere ottenute dall'analisi in modalità QI. In particolare, l'analisi dei dati QI consente di valutare il modulo di Young del campione. Le Fig. 4b,e mostrano le mappe 2D dei valori del modulo di Young relativi agli esperimenti, rispettivamente, a bassa ed alta forza ionica. Come previsto, il substrato d'oro presenta un modulo di Young decisamente più elevato rispetto al film di DNA nel caso di SAM preparati ad alta forza ionica (Fig. 4e) mentre a bassa concentrazione di sale (Fig. 4b) non vi è una differenza significativa tra substrato e SAM. Quando presente, la differenza nei valori dei moduli di Young del film e del substrato può essere meglio apprezzata analizzando gli istogrammi delle mappe dei moduli di Young riportate nelle Fig. 4c,f. L'istogramma in Fig. $4 c$ può essere riprodotto con un singolo profilo gaussiano centrato a $100 \mathrm{MPa}$, il che significa che nel caso di SAM depositati a bassa forza ionica non è possibile rilevare alcuna differenza significativa tra il modulo di Young del substrato nudo e del substrato coperto dal SAM. Viceversa, ad alta forza ionica, devono essere utilizzati due profili gaussiani per riprodurre l'inviluppo dell'istogramma (Fig. 4f). Dall'analisi 
dei dati risulta una differenza di circa $40 \mathrm{Mpa}$ tra il centro del profilo gaussiano che corrisponde al SAM (curva marrone, centrata a $57 \mathrm{MPa}$ ) e il centro del profilo gaussiano che corrisponde al substrato (curva gialla, centrata a $100 \mathrm{Mpa}$ ). I valori del modulo di Young del biofilm risultano, come previsto, inferiori a quelli del substrato d'oro.

\section{bassa forza ionica}
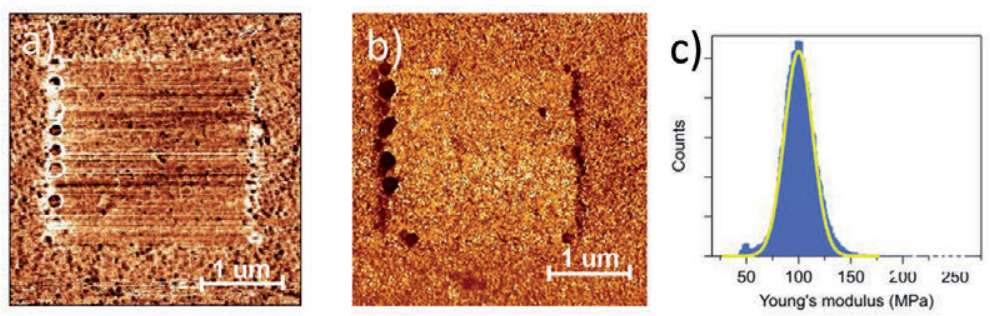

\section{alta forza ionica}
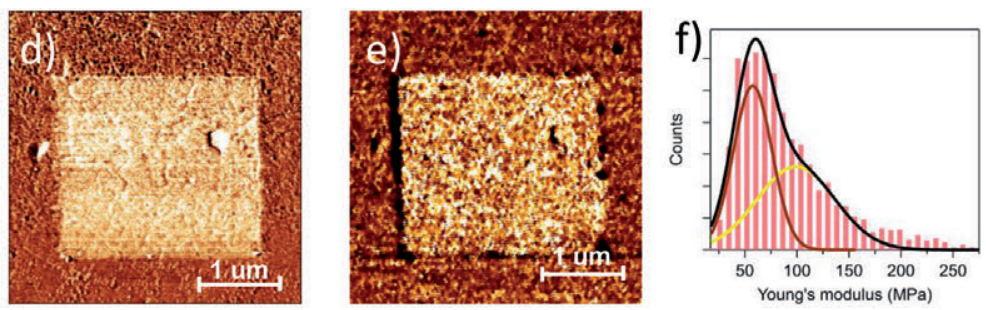

Fig. 4. Esperimenti di shaving su SAM di DNA preparati a bassa forza ionica (a-c) e ad alta forza ionica $(d-f) .(a, d)$ Immagini in deflessione laterale acquisite in modalità contatto (scala: $20 \mathrm{mV}$ ). (b,e) Mappe del modulo di Young ottenute dall' analisi delle curve di forza in QI (scala: $150 \mathrm{Mpa}$ ) e (c,f) relativi istogrammi. Modificata da [38].

Gli esperimenti di nanoshaving indicano che la forza ionica del tampone influenza fortemente la deposizione del DNA. A bassa forza ionica, a causa della repulsione tra i filamenti di DNA, carichi negativamente, si ottiene un SAM sottile, di bassa densità verosimilmente formato da molecole quasi sdraiate che assumono una conformazione prevalentemente allungata. Viceversa, ad alta forza ionica, la carica del DNA risulta schermata con una conseguente conformazione più ripiegata e una ridotta repulsione tra filamenti adiacenti. In queste condizioni si ottiene un SAM più spesso e con una copertura maggiore formata 
da molecole quasi "in piedi". Questa interpretazione è in accordo con l'osservazione, su SAM di densità molecolare fissata, di filamenti allungati a bassa forza ionica e filamenti ripiegati ad alta forza ionica [39].
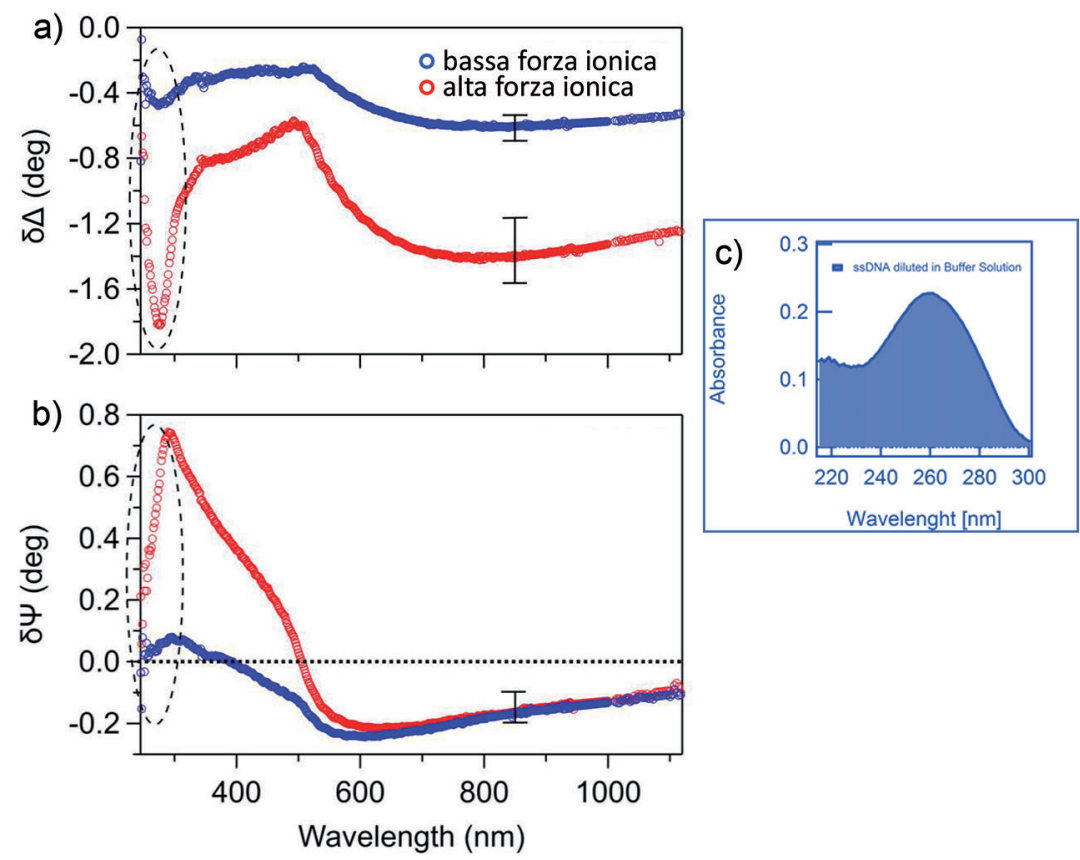

Fig. 5. (a,b) Spettri ellissometrici differenza relativi a SAM depositati a bassa forza ionica (curve blu) e ad alta forza ionica (curve rosse). Le regioni evidenziate indicano $i$ minimi associati all'assorbimento molecolare del DNA. (c) Assorbimento UV-Vis di una soluzione di DNA. Modificata da [38].

Una conferma dei risultati AFM viene dall'analisi spettroellissometrica (SE). In Fig. 5 sono riportati gli spettri differenza ottenuti sottraendo agli spettri acquisiti dopo la deposizione del SAM gli spettri del solo substrato. L'analisi degli spettri differenza consente di mettere in evidenza il contributo del SAM. Per una discussione più dettagliata di questi dati si rimanda alla ref. [38]. Una prima caratteristica spettrale interessante delle curve SE è la presenza di un minimo profondo intorno a $270 \mathrm{~nm}$ in $\delta \Delta$ (Fig. 5a) e di una corrispondente decrescita di $\delta \Psi$ intorno a $290 \mathrm{~nm}$ (Fig. 5b). Questi minimi sono ben definiti ad alta forza ionica (curve rosse), ma sono presenti, seppure più deboli, anche 
a bassa forza ionica (curve blu). Considerando che l'assorbimento UVVis del DNA in soluzione presenta una banda di assorbimento situata a $260 \mathrm{~nm}$ (Fig. 5c) e che i film trasparenti presentano un comportamento molto diverso in questa regione [40], è ragionevole attribuire questa struttura spettrale all'assorbimento del DNA. Va inoltre osservato che i valori di I $\delta \Delta \mid$ nel NIR (lontano dalle risonanze molecolari) e i valori $\delta \Psi$ nel vicino UV sono decisamente maggiori per i SAM depositati ad elevata forza ionica. Come discusso in precedenti lavori [30,41], queste caratteristiche sono associate allo spessore ottico del film, una grandezza correlata all'indice di rifrazione e allo spessore del film, che risulta più elevata per i SAM depositati ad elevata forza ionica. I dati SE confermano quindi che la forza ionica del buffer gioca un ruolo significativo nella formazione del SAM. Va notato, inoltre, che per i film ultrasottili, come i monostrati analizzati in questo studio, l'indice di rifrazione e lo spessore sono parametri altamente correlati. Gli esperimenti di nanoshaving possono essere sfruttati per risolvere questa correlazione: assumendo come valori per lo spessore del SAM i risultati ottenuti dall'AFM, è infatti possibile ricavare una stima dell'indice di rifrazione attraverso un confronto tra dati ellissometrici e curve simulate ottenute a partire da un opportuno modello ottico del sistema. Questo tipo di analisi, per i cui dettagli si rimanda alla ref. [38], fornisce, per i SAM depositati ad elevata forza ionica, valori dell'indice di rifrazione intorno a 1.45 per $\lambda=650 \mathrm{~nm}$, valore in ragionevole accordo con la formazione di SAM compatti.

\section{CONCLUSIONI}

L'accoppiamento di nanolitografia AFM e spettroellissometria ha permesso di caratterizzare le proprietà morfologiche, meccaniche ed ottiche di SAM di DNA e di valutarne la dipendenza dalla forza ionica della soluzione utilizzata per la deposizione dei SAM stessi. I SAM depositati ad elevata forza ionica risultano più spessi, densi e meno deformabili rispetto a quelli depositati a bassa forza ionica. In particolare, sui SAM densi, l'utilizzo dell'AFM in modalità QI ha consentito di rilevare una differenza nel modulo di Young tra SAM e substrato. Inoltre, l'analisi degli spettri ellissometrici ha permesso di rilevare l'assorbimento molecolare del DNA a livello di singolo monostrato. 


\section{BIBLIOGRAFIA}

1. Bain, C.D.; Whitesides, G.M. Molecular-Level Control over Surface Order in SelfAssembled Monolayer Films of Thiols on Gold. Science 1988, 240, 62-63, doi:10.1126/science.240.4848.62.

2. Nuzzo, R.G.; Zegarski, B.R.; Dubois, L.H. Fundamental studies of the chemisorption of organosulfur compounds on gold(111). Implications for molecular selfassembly on gold surfaces. J. Am. Chem. Soc. 1987, 109, 733-740, doi:10.1021/ ja00237a017.

3. Bain, C.D.; Troughton, E.B.; Tao, Y.T.; Evall, J.; Whitesides, G.M.; Nuzzo, R.G. Formation of monolayer films by the spontaneous assembly of organic thiols from solution onto gold. J. Am. Chem. Soc. 1989, 111, 321-335, doi:10.1021/ja $00183 \mathrm{a} 049$.

4. Love, J.C.; Estroff, L.A.; Kriebel, J.K.; Nuzzo, R.G.; Whitesides, G.M. SelfAssembled Monolayers of Thiolates on Metals as a Form of Nanotechnology. Chem. Rev. 2005, 105, 1103-1170, doi:10.1021/cr0300789.

5. Pujari, S.P.; Scheres, L.; Marcelis, A.T.M.; Zuilhof, H. Covalent Surface Modification of Oxide Surfaces. Angew. Chem. Int. Ed. 2014, 53, 6322-6356, doi:10. 1002/anie.201306709.

6. Flink, S.; Veggel, F.C.J.M. van; Reinhoudt, D.N. Sensor Functionalities in SelfAssembled Monolayers. Advanced Materials 2000, 12, 1315-1328, doi:10.1002/1521-4095(200009)12:18<1315::AID-ADMA1315>3.0.CO;2-K.

7. Ibau, C.; Md Arshad, M.K.; Gopinath, S.C.B.; Nuzaihan M.N, M.; M. Fathil, M.F.; Estrela, P. Gold interdigitated triple-microelectrodes for label-free prognosticative aptasensing of prostate cancer biomarker in serum. Biosensors and Bioelectronics 2019, 136, 118-127, doi:10.1016/j.bios.2019.04.048.

8. Yu, S.; Chen, T.; Zhang, Q.; Zhou, M.; Zhu, X. Application of DNA nanodevices for biosensing. Analyst 2020, 145, 3481-3489, doi:10.1039/D0AN00159G.

9. MacAskill, A.; Crawford, D.; Graham, D.; Faulds, K. DNA Sequence Detection Using Surface-Enhanced Resonance Raman Spectroscopy in a Homogeneous Multiplexed Assay. Anal. Chem. 2009, 81, 8134-8140, doi:10.1021/ac901361b.

10. Qian, S.; Lin, M.; Ji, W.; Yuan, H.; Zhang, Y.; Jing, Z.; Zhao, J. Boronic Acid Functionalized Au Nanoparticles for Selective MicroRNA Signal Amplification in Fiber-Optic Surface Plasmon Resonance Sensing System. ACS Sensors 2018, 7.

11. Caneira, C.R.F.; Soares, R.R.G.; Pinto, I.F.; Mueller-Landau, H.S.; Azevedo, A.M.; Chu, V.; Conde, J.P. Development of a rapid bead-based microfluidic platform for DNA hybridization using single- and multi-mode interactions for probe immobilization. Sensors and Actuators B: Chemical 2019, 286, 328-336, doi:10.1016/ j.snb.2019.01.133.

12. Teengam, P.; Siangproh, W.; Tuantranont, A.; Vilaivan, T.; Chailapakul, O.; Henry, C.S. Multiplex Paper-Based Colorimetric DNA Sensor Using Pyrrolidinyl Peptide Nucleic Acid-Induced AgNPs Aggregation for Detecting MERS-CoV, MTB, and HPV Oligonucleotides. Anal. Chem. 2017, 89, 5428-5435, doi:10.1021/acs.analchem.7b00255.

13. Kim, H.; Park, M.; Hwang, J.; Kim, J.H.; Chung, D.-R.; Lee, K.; Kang, M. 
Development of Label-Free Colorimetric Assay for MERS-CoV Using Gold Nanoparticles. ACS Sens. 2019, 4, 1306-1312, doi:10.1021/acssensors.9b00175.

14. Wold, E.D.; McBride, R.; Axup, J.Y.; Kazane, S.A.; Smider, V.V. Antibody Microarrays Utilizing Site-Specific Antibody-Oligonucleotide Conjugates. Bioconjugate Chem. 2015, 26, 807-811, doi:10.1021/acs.bioconjchem.5b00111.

15. Yang, Y.; Wang, S.; Zhou, Z.; Zhang, R.; Shen, H.; Song, J.; Su, P.; Yang, Y. Enhanced reusability and activity: DNA directed immobilization of enzyme on polydopamine modified magnetic nanoparticles. Biochemical Engineering Journal 2018, 137, 108-115, doi:10.1016/j.bej.2018.05.019.

16. Wang, H.; Liu, M.; Bai, W.; Sun, H.; Li, Y.; Deng, H. A convenient electrogenerated chemiluminescence biosensing method for selective detection of 5-hydroxymethylcytosine in genomic DNA. Sensors and Actuators B: Chemical 2019, 284, 236-242, doi:10.1016/j.snb.2018.12.132.

17. Herne, T.M.; Tarlov, M.J. Characterization of DNA Probes Immobilized on Gold Surfaces. Journal of the American Chemical Society 1997, 119, 8916-8920, doi:10.1021/ja9719586.

18. Liu, D.; Bruckbauer, A.; Abell, C.; Balasubramanian, S.; Kang, D.-J.; Klenerman, D.; Zhou, D. A Reversible pH-Driven DNA Nanoswitch Array. Journal of the American Chemical Society 2006, 128, 2067-2071, doi:10.1021/ja0568300.

19. Guiducci, C.; Stagni, C.; Fischetti, A.; Mastromatteo, U.; Benini, L.; Riccoricco, B. Microelectrodes on a Silicon Chip for Label-Free Capacitive DNA Sensing. IEEE Sensors J. 2006, 6, 1084-1093, doi:10.1109/JSEN.2006.877979.

20. Drozd, M.; Pietrzak, M.D.; Malinowska, E. SPRi-Based Biosensing Platforms for Detection of Specific DNA Sequences Using Thiolate and Dithiocarbamate Assemblies. Front Chem 2018, 6, doi:10.3389/fchem.2018.00173.

21. Li, Z.; Niu, T.; Zhang, Z.; Feng, G.; Bi, S. Effect of monovalent cations ( $\mathrm{Li}+, \mathrm{Na}+$, $\mathrm{K}+, \mathrm{Cs}+)$ on self-assembly of thiol-modified double-stranded and single-stranded DNA on gold electrode. Analyst 2012, 137, 1680-1691, doi:10.1039/c2an15716k.

22. Guilbaud, S.; Salomé, L.; Destainville, N.; Manghi, M.; Tardin, C. Dependence of DNA Persistence Length on Ionic Strength and Ion Type. Phys. Rev. Lett. 2019, 122, 028102, doi:10.1103/PhysRevLett.122.028102.

23. Moses, S.; Brewer, S.H.; Lowe, L.B.; Lappi, S.E.; Gilvey, L.B.G.; Sauthier, M.; Tenent, R.C.; Feldheim, D.L.; Franzen, S. Characterization of Single- and DoubleStranded DNA on Gold Surfaces. Langmuir 2004, 20, 11134-11140, doi:10.1021/ la0492815.

24. Keighley, S.D.; Li, P.; Estrela, P.; Migliorato, P. Optimization of DNA immobilization on gold electrodes for label-free detection by electrochemical impedance spectroscopy. Biosensors and Bioelectronics 2008, 23, 1291-1297, doi:10.1016/j.bios. 2007.11.012.

25. Wolf, L.K.; Gao, Y.; Georgiadis, R.M. Sequence-Dependent DNA Immobilization: Specific versus Nonspecific Contributions. Langmuir 2004, 20, 3357-3361, doi:10.1021/la036125+.

26. Petrovykh, D.Y.; Kimura-Suda, H.; Whitman, L.J.; Tarlov, M.J. Quantitative Analysis and Characterization of DNA Immobilized on Gold. Journal of the American Chemical Society 2003, 125, 5219-5226, doi:10.1021/ja029450c. 
27. Howell, C.; Hamoudi, H.; Zharnikov, M. Thymine/adenine diblock-oligonucleotide monolayers and hybrid brushes on gold: a spectroscopic study. Biointerphases 2013, 8, 6, doi:10.1186/1559-4106-8-6.

28. Charrier, E.E.; Pogoda, K.; Wells, R.G.; Janmey, P.A. Control of cell morphology and differentiation by substrates with independently tunable elasticity and viscous dissipation. Nat Commun 2018, 9, 449, doi:10.1038/s41467-018-02906-9.

29. Solano, I.; Parisse, P.; Gramazio, F.; Cavalleri, O.; Bracco, G.; Castronovo, M.; Casalis, L.; Canepa, M. Spectroscopic ellipsometry meets AFM nanolithography: about hydration of bio-inert oligo(ethylene glycol)-terminated self assembled monolayers on gold. Physical Chemistry Chemical Physics 2015, 17, 28774-28781, doi:10.1039/C5CP04028K.

30. Pinto, G.; Parisse, P.; Solano, I.; Canepa, P.; Canepa, M.; Casalis, L.; Cavalleri, O. Functionalizing gold with single strand DNA: novel insight into optical properties via combined spectroscopic ellipsometry and nanolithography measurements. Soft Matter 2019, 15, 2463-2468, doi:10.1039/C8SM02589D.

31. Xu, S.; Liu, G.-Y. Nanometer-Scale Fabrication by Simultaneous Nanoshaving and Molecular Self-Assembly. Langmuir 1997, 13, 127-129, doi:10.1021/la962029f.

32. Liu, M.; Amro, N.A.; Chow, C.S.; Liu, G. Production of Nanostructures of DNA on Surfaces. Nano Letters 2002, 2, 863-867, doi:10.1021/nl025626x.

33. Liu, S.; Maoz, R.; Schmid, G.; Sagiv, J. Template Guided Self-Assembly of [Au55] Clusters on Nanolithographically Defined Monolayer Patterns. Nano Lett. 2002, 2, 1055-1060, doi:10.1021/nl025659c.

34. Unruh, D.A.; Malduin, C.; Pastine, S.J.; Rolandi, M.; Fréchet, J.M.J. Bifunctional Patterning of Mixed Monolayer Surfaces Using Scanning Probe Lithography for Multiplexed Directed Assembly. Journal of the American Chemical Society 2010, 132, 6890-6891, doi:10.1021/ja101627e.

35. Ambrosetti, E.; Paoletti, P.; Bosco, A.; Parisse, P.; Scaini, D.; Tagliabue, E.; de Marco, A.; Casalis, L. Quantification of Circulating Cancer Biomarkers via Sensitive Topographic Measurements on Single Binder Nanoarrays. ACS Omega 2017, 2, 2618-2629, doi:10.1021/acsomega.7b00284.

36. Solano, I.; Parisse, P.; Gramazio, F.; Ianeselli, L.; Medagli, B.; Cavalleri, O.; Casalis, L.; Canepa, M. Atomic Force Microscopy and Spectroscopic Ellipsometry combined analysis of Small Ubiquitin-like Modifier adsorption on functional monolayers. Applied Surface Science 2017, 421, 722-727, doi:10.1016/j.apsusc.2016.10.195.

37. Dufrêne, Y.F.; Martínez-Martín, D.; Medalsy, I.; Alsteens, D.; Müller, D.J. Multiparametric imaging of biological systems by force-distance curve-based AFM. Nat Methods 2013, 10, 847-854, doi:10.1038/nmeth.2602.

38. Pinto, G.; Canepa, P.; Canale, C.; Canepa, M.; Cavalleri, O. Morphological and Mechanical Characterization of DNA SAMs Combining Nanolithography with AFM and Optical Methods. Materials 2020, 13, 2888, doi:10.3390/ma13132888.

39. Gil, P.S.; Lacks, D.J.; Parisse, P.; Casalis, L.; Nkoua Ngavouka, M.D. Single-stranded DNA oligomer brush structure is dominated by intramolecular interactions mediated by the ion environment. Soft Matter 2018, 14, 9675-9680, doi:10.1039/C8SM01743C.

40. Prato, M.; Moroni, R.; Bisio, F.; Rolandi, R.; Mattera, L.; Cavalleri, O.; Canepa, M. 
Optical Characterization of Thiolate Self-Assembled Monolayers on $\mathrm{Au}(111)$. The Journal of Physical Chemistry C 2008, 112, 3899-3906, doi:10.1021/jp711194s.

41. Parisse, P.; Solano, I.; Magnozzi, M.; Bisio, F.; Casalis, L.; Cavalleri, O.; Canepa, M. Thickness and Beyond. Exploiting Spectroscopic Ellipsometry and Atomic Force Nanolithography for the Investigation of Ultrathin Interfaces of Biologic Interest. In Ellipsometry of Functional Organic Surfaces and Films; Hinrichs, K., Eichhorn, K.-J., Eds.; Springer International Publishing: Cham, 2018; Vol. 52, pp. 63-93 ISBN 978-3-319-75894-7. 
